\title{
Magnetic Field Influence on Electrical Properties of Human Blood Measured by Impedance Spectroscopy
}

\author{
M. Sosa, ${ }^{1 *}$ J. Bernal-Alvarado, ${ }^{1}$ M. Jiménez-Moreno, ${ }^{1}$ J.C. Hernández, ${ }^{1}$ G. Gutiérrez-Juárez, \\ M. Vargas-Luna, ${ }^{1}$ R. Huerta, ${ }^{2}$ J.C. Villagómez-Castro, ${ }^{3}$ and P. Palomares ${ }^{4}$ \\ ${ }^{1}$ Instituto de Física, Universidad de Guanajuato, León, Gto., México \\ ${ }^{2}$ Instituto de Investigación Sobre el Trabajo, Universidad de Guanajuato, León, \\ Gto., México \\ ${ }^{3}$ Instituto de Investigaciones en Biología Experimental, Facultad de Química, \\ Universidad de Guanajuato, Guanajuato, Gto., México \\ ${ }^{4}$ Centro Estatal de Transfusión Sanguínea, León, Gto., México
}

\begin{abstract}
The impedance spectroscopy technique (IST) was used for studying the effect of a $0.5 \mathrm{~T}$ magnetic field on the electrical properties of whole human blood. A Solartron SI 1260 spectrometer was used to measure the impedance spectra of magnetic field exposed blood samples compared to non-exposed samples. An equivalent electrical circuit model, consisting in a resistance $R_{\mathrm{S}}$ in series with a parallel circuit formed by a constant phase element (CPE) and another resistance $R_{\mathrm{p}}$, is proposed to fit the data in both cases. The experiment used $3 \mathrm{ml}$ human blood samples from 160 healthy donors. A Wilcoxon matched pairs statistical test was applied to the data. The data analysis seems to show a statistically significant increase of the values of resistance $R_{\mathrm{p}}(Z=5.06, P<0.001)$ and capacitance $C_{\mathrm{T}}(Z=3.32$, $P<0.001)$ of the blood exposed to magnetic field, by approximately $10.4 \%$ and $1.9 \%$, respectively. Bioelectromagnetics 26:564-570, 2005. ㅇ 2005 Wiley-Liss, Inc.
\end{abstract}

Key words: static magnetic field; electrical parameters

\section{INTRODUCTION}

The impedance spectroscopy technique (IST) has been commonly used to determine the electrical characteristics of a wide variety of materials. The principle of IST is based on the frequency response of the material when an harmonic voltage is applied [McDonald, 1987]. Although biological materials are very complex to characterize due to their inherent variability, many investigations have reported the use of IST for the study of biological materials and processes.

Blood properties have been widely investigated using spectroscopic techniques. Reviews related to impedance spectroscopy of biological tissues (blood included), can be found elsewhere [Gabriel et al., 1996a]. The physical parameters of the equivalent circuits are also tabulated for specific electrode geometries and bandwidths from the $\beta$ to $\delta$ dielectric dispersions [Gabriel et al., 1996b]. Also, studies of dielectric properties of human blood have been conducted at different frequency ranges, such as microwave frequencies [Alison and Sheppard, 1993] and radio frequencies [Beving et al., 1994].

Very recently Jaspard and Nadi [2002] have reported a study of the dielectric properties of blood as a function of temperature. They found a weak dependence of the permittivity and conductivity as a function of temperature variations. More recently Jaspard et al. [2003] have also published a study of the dielectric properties of blood as a function of the hematocrit percentage, where they found a strong dependence on the concentration. In these works a frequency range from $1 \mathrm{MHz}$ to $1 \mathrm{GHz}$ was swept, and measurements were performed on blood ex vivo of humans, cows, and sheep.

Time domain dielectric spectroscopy studies of erythrocyte cells have been performed by Lisin et al. [1996], who estimated the dielectric constants of cell

Grant sponsor: CONACyT; Grant number: 38749-E.

*Correspondence to: M. Sosa, Instituto de Física, Universidad de Guanajuato, Apartado Postal E-143, 37000 León, Gto., México. E-mail: modesto@ fisica.ugto.mx

Received for review 13 September 2004; Final revision received 4 March 2005

DOI 10.1002/bem.20132

Published online 2 September 2005 in Wiley InterScience (www.interscience.wiley.com). 
structural parts, such as membrane and cytoplasm. Also, Polevaya et al. [1999] studied time domain dielectric spectroscopy of normal and malignant white blood cells. They found differences in the dielectric permittivity, capacitance, and conductivity values of cell membranes for normal and malignant lymphocytes.

Particularly interesting are the works by Zhao [1993] and Zhao et al. [1993], who investigated the relationship between the electrical parameters and the human blood hematocrit for four types of anticoagulant (including EDTA), finding differences in the electrical parameters among samples with various anticoagulants. Zhao et al. [1993] performed detailed measurements of plasma resistance, cell interior resistance, and cell membrane capacitance of blood. In this work, the authors proposed a three-element circuit model for simulating the blood impedance, which was found feasible.

Several approaches have been proposed to model the blood dielectric properties. Grosse [1998] used a simple counterion model, in which the fluid is treated as a suspension of cells inside an electrolyte solution (the plasma). Also, Grosse and Schwan [1992] have proposed that a microscopic model of potentials induced by alternating electrical fields, applied on cells, must include additional information such as membrane conductance and surface admittance. A different approach based on the Maxwell-Wagner model has been used for analyzing the dielectric properties of suspensions of erythrocytes [Beving and Eriksson, 1994; Hayashi et al., 2003].

On the other hand, since the pioneer work by Pauling and Coryell [1936] on the magnetic susceptibility of blood, many investigations have reported both the magnetic properties and the influence of an external magnetic field on blood. Pauling and Coryell [1936] studied the magnetic properties and the structure of the hemoglobin. They were the first to report the diamagnetic susceptibility of the oxyhemoglobin and the paramagnetic susceptibility of the deoxyhemoglobin.

Higashi et al. [1993] studied the orientation of erythrocytes in an $8 \mathrm{~T}$ static magnetic field. They found that erythrocytes are oriented with the plane of their disc parallel to the direction of the magnetic field. Yamagishi et al. [1992] observed diamagnetic orientation of red blood cells under the application of a high magnetic field. Shalygin et al. [1983] studied the behavior of erythrocytes under strong magnetic field gradients. They reported a susceptibility for diamagnetic erythrocytes of $-(0.13-0.65) \times 10^{-8} \mathrm{cgs} \mathrm{emu} / \mathrm{cm}^{3}$ Oe and $(13-33) \times 10^{-8} \mathrm{cgs} \mathrm{emu} / \mathrm{cm}^{3}$ Oe for paramagnetic erythrocytes. Nakano et al. [1972] have also reported the effect of a magnetic field on the erythrocyte rotation. The influence of alternating electric currents on human blood has been also investigated by Poppendiek and Hody [1963], Poppendiek et al. [1964], and more recently by De Vries et al. [1995]. Other investigations have been performed to study aspects such as the osmotic fragility of the erythrocyte under a magnetic field [Coldman et al., 1969].

Very recently, Yamamoto et al. [2004] examined the mechanisms of the magnetic field influence on blood viscosity by measuring the time for blood to fall inside an $1.5 \mathrm{~T}$ static magnetic field. They measured a significant increase on blood viscosity. Also, Capri et al. [2004] studied the effects of $50 \mathrm{~Hz}$ sinusoidal magnetic fields on human lymphocytes. They observed no effects on lymphocyte activation and proliferation.

In this study, IST was used to determine the influence of a $0.5 \mathrm{~T}$ static magnetic field on the electrical properties of human blood. So, the aim of this investigation was to demonstrate the feasibility of using a physical measurement provided by IST for hematology research, in particular to get information on how the magnetic field influences the human blood.

\section{THEORETICAL CONCERNS}

\section{Blood}

Red cells contain hemoglobin, which is an ironcontaining protein capable of adding oxygen molecules to itself. The molecular structural configuration of the hemoglobin strongly depends on the presence of oxygen. The hemoglobin containing oxygen is called oxyhemoglobin; otherwise, it is called deoxyhemoglobin. These structural changes are responsible, among other factors, for its magnetic behavior [Pauling and Coryell, 1936]. The oxyhemoglobin has been observed to exhibit a diamagnetic behavior, while the deoxyhemoglobin has a paramagnetic susceptibility.

Also, we have to note that a widely accepted physical mechanism to explain the impedance behavior of blood is based on ionic currents through the interstitial medium. This picture takes into account the highly non-conductive characteristics of the lipid cellular membrane, so that ions have to move preferentially in the bulk medium and around the cellular surface. In light of this, the resistance to the ionic current comes from both the charge density at the cellular membrane and the presence of ions in the bulk.

\section{Electrical Model $\boldsymbol{R}_{s}$ [CPE- $\left.\boldsymbol{R}_{\mathrm{p}}\right]$}

The analysis we proposed for the electrical characterization of blood consisted of its frequency response when an harmonic voltage was applied. Despite IST being an experimental method used for decades for this purpose, a significant development has 
occurred for the theoretical model proposed by Cole and Cole [1941]. This new model generalizes the expressions for the equivalent impedance of an $\mathrm{RC}$ circuit to the case in which the currents in the capacitive medium have a distribution of time constants [McDonald, 1987]. Hence, in many biological systems a constant phase element (CPE) is frequently proposed to model the data instead of a capacitor. The impedance $Z_{\mathrm{CPE}}$ of a CPE element is given by

$$
Z_{\mathrm{CPE}}=\frac{1}{(j \omega)^{P} C_{\mathrm{T}}},
$$

where $j=(-1)^{1 / 2}, \omega$ is the angular frequency, and $C_{\mathrm{T}}$ and $P$ are parameters which describe the CPE. It is important to emphasize that, strictly speaking, dimensionally $\left[C_{\mathrm{T}}\right]=\mathrm{F} \cdot \mathrm{s}^{P-1}$, where $\mathrm{F}$ and $\mathrm{s}$ refer to Farads and seconds, respectively. However, in this work the value of $P$ was nearly 1 , in fact $P=0.97$ on average. Hence, for the sake of simplicity $C_{\mathrm{T}}$ will be considered throughout this work as a capacitance.

Therefore, an electrical model $R_{\mathrm{s}}\left[\mathrm{CPE}-R_{\mathrm{p}}\right]$, consisting of a resistance $R_{\mathrm{S}}$ in series with a parallel circuit formed by a CPE and another resistance $R_{\mathrm{p}}$, gives a first approach to a physical model of the human blood electrical behavior in our experiment. The total impedance of the proposed model is

$$
Z_{\mathrm{TOTAL}}=R_{\mathrm{S}}+\frac{R_{\mathrm{p}}}{1+R_{\mathrm{p}}(j \omega)^{\mathrm{P}} C_{\mathrm{T}}},
$$

where the resistance $R_{\mathrm{S}}$ is introduced to take into account the intrinsic contribution of the measurement system and the interface between the sample and the electrodes, while the CPE- $R_{\mathrm{p}}$ element characterizes the electrical properties of the sample.

\section{MATERIALS AND METHODS}

An impedance/gain-phase analyzer Solartron SI 1260 (Solartron Analytical, Houston, TX) was used to determine the electrical impedance spectra of whole human blood samples [Richert, 1996]. The spectrometer has a working frequency range from $1 \mu \mathrm{Hz}$ to $32 \mathrm{MHz}$, and its accuracy has been tested in several applications. In this experiment, a frequency range from $1 \mathrm{~Hz}$ and $10 \mathrm{KHz}$ was swept, applying a polarization voltage of $10 \mathrm{mV}$. This experimental setup permits the detection of the blood $\beta$ relaxation process. Other dielectric processes such as $\delta$ dispersion are not completely observed with this system.

\section{Electrodes}

Blood glucose test strips (Bayer ${ }^{\mathrm{TM}}$ ) were used as electrodes, instead of the conventional metallic ones.
These test strips allow gathering $2 \mu 1$ of sample to be analyzed by the spectrometer. Their use as electrodes was carefully tested in order to determine what kind of chemical reaction occurs between blood and test strips, as well as the evolution in time of the reaction, and its possible influence on the impedance measurements. Tests performed included monitoring of stability of the spectrometer output signal. An average time of $7 \mathrm{~min}$ was determined to be necessary to have a significant change on the signal. This value agrees with that given by the manufacturer, which is between 8 and $10 \mathrm{~min}$. In our experiment, the measurement time was less than $1 \mathrm{~min}$, to assure that the effect on the impedance could be neglected. We also want to emphasize that the use of test strips as electrodes was motivated by several facts: First, these are disposable, a new strip was used for any measurement. Second, their manufacture is commercially standardized.

\section{Samples}

One hundred sixty whole human blood samples of $3 \mathrm{ml}$ from healthy subjects were measured. Donors were adult subjects from $18-54$ years old (mean $32.0 \pm$ 8.3 years), 29 women, and 131 men. Samples were in the hematocrit range of $40.4 \%-54.8 \%$ (mean $47.7 \pm$ $2.8 \%$ ). A previous screen was performed on all samples at the Centro Estatal de Transfusión Sanguínea at Guanajuato, to discard transmissible bacterial and viral diseases. The samples were provided in $5 \mathrm{ml}$ vacutainers (Golden Hour Medical, Brooklyn, NY, USA), which contain a minimum quantity of ethylenediaminetetraacetic acid (EDTA), less than $1 \%$ in volume, added to prevent coagulation. The measurement protocol was approved by the ethics committee from the University of Guanajuato and was according to the Declaration of Helsinki. All subjects enrolled in the study gave their informed consent.

\section{Procedure}

The samples were first measured without magnetic exposure and their electrical parameters registered. The measurements were repeated under the influence of a $0.5 \mathrm{~T}$ static magnetic field produced by an arrangement of rare earth permanent magnets. $\mathrm{NdFeB}$ permanent magnets of $2.5 \times 2.5 \times 1.3 \mathrm{~cm}$ sides (Chen Yang Magnetics, Erding, Germany), with a magnetic moment $m=0.78 \pm 0.05 \mathrm{~A} \times \mathrm{m}^{2}$ were used. A 2100 model gaussmeter (Magnetic Instrumentation, Inc., Indianapolis, IN, USA) was used to measure the magnetic field. When the magnetic field was applied, the arrangement of permanent magnets was located just under the vacutainers. The magnetic field was measured placing the probe on the bottom of the vacutainer. The whole system was on a non-magnetic 


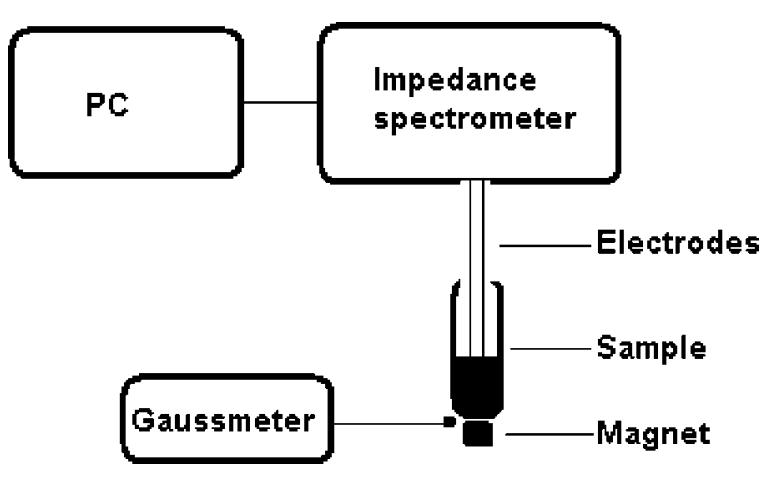

Fig. 1. Schematic diagram of the experimental setup.

table. Figure 1 shows a schematic diagram of the experimental setup.

Twenty samples were measured per day, both outside the magnetic field and during magnetic field exposure. The measurement of one sample last about $1 \mathrm{~min}$. Hence, there was an interval of $20 \mathrm{~min}$ between measurements of the same sample in both conditions. Assessments were performed at room temperature $\left(25 \pm 1{ }^{\circ} \mathrm{C}\right)$.

Some tests were made in order to determine if the time between measurements makes a difference on the results. First, two sets of samples were measured only once, either without magnetic field or under magnetic field exposure. Second, some measurements were made by applying the magnetic field during different time intervals. For example, a measurement was performed immediately after applying the magnetic field; a second was made after 15 min of magnetic field exposure and still in presence of the magnetic field, and a third was performed 15 min after removing the magnets. In this last case, the samples were first kept 15 min under magnetic field. Data analysis of these tests shows general features in agreement with those in the first experimental procedure.

Also, some tests were performed to determine the effect of the magnetic field on electrodes. An electrode reference signal was registered without the magnetic field and under magnetic field. No significant changes were observed in its response.

\section{RESULTS AND DISCUSSION}

By using the experimental methodology described above, measurements of the impedance parameters and hence the possible effects of a magnetic field on the blood electrical characteristics were performed.

In Figure 2, the characteristic spectra for a typical blood sample obtained using IST are shown. No significant differences in the functional shape of the spectra were observed due to the presence of the magnetic field. The same behavior shown in Figure 2 was observed
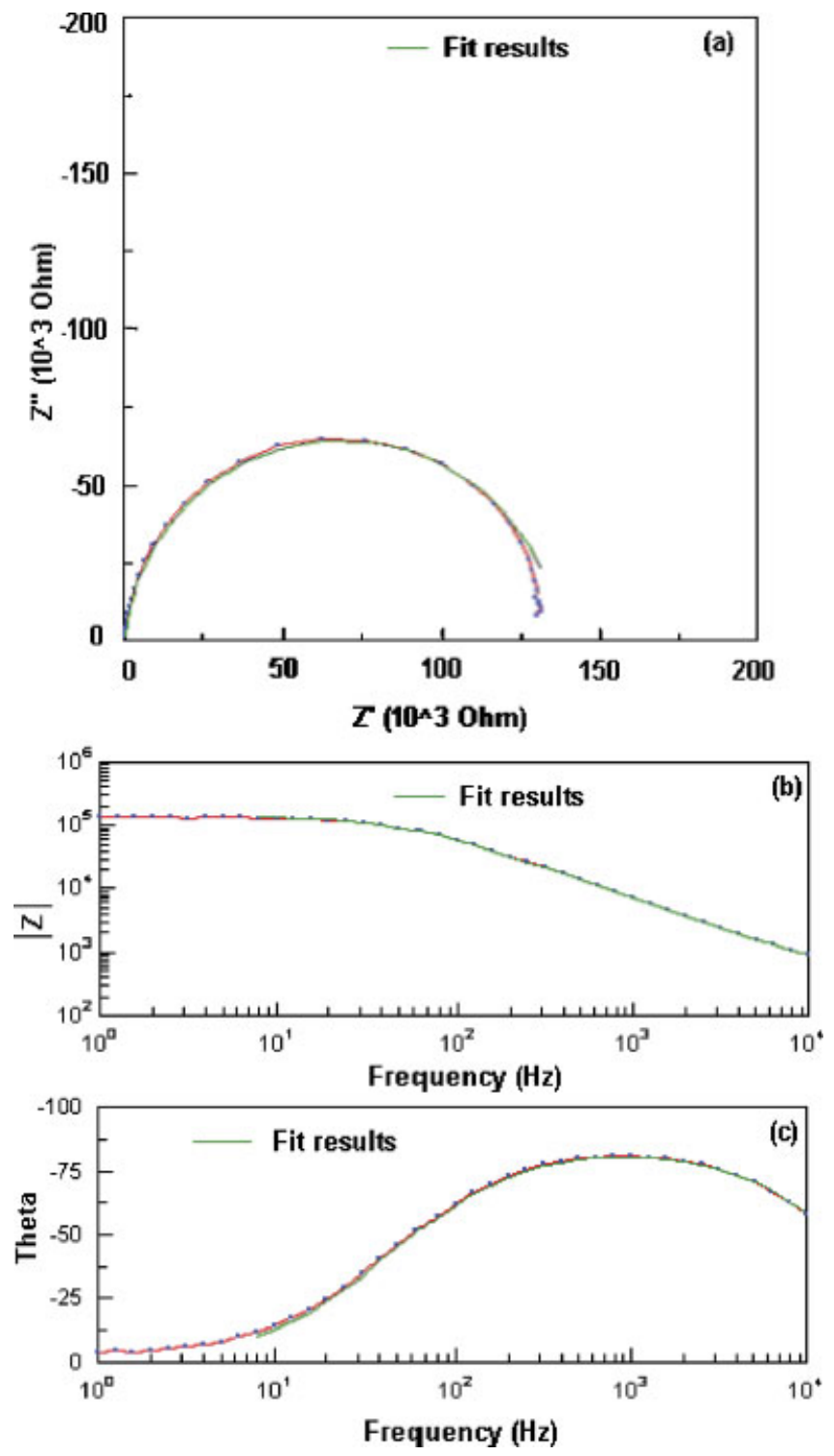

Fig. 2. Characteristic spectra for a typical blood sample. a: Representation on the complex plane, $Z^{\prime \prime}$ versus $Z^{\prime}$, of the impedance obtained at a $10 \mathrm{mV}$ polarization voltage. $\mathbf{b}$ : Impedance amplitude versus frequency. c: Phase versus frequency. The continuous line represents the fit of a $R_{\mathrm{s}}\left[\mathrm{CPE}-R_{\mathrm{p}}\right]$ model to the data. A non-linear least-squares (NLLS) algorithm was used for fitting. [The color figure for this article is available online at www. interscience. wiley. com.]

for all the samples. An implementation of the nonlinear least-squares (NLLS) Marquardt-Levenberg algorithm, in the Z-view software of the Solartron spectrometer, was used to fit the electrical model proposed to the experimental data. In this figure, the $\beta$ relaxation process is clearly observed.

Figure 3 shows a comparison between the distributions of blood electrical parameters from the samples without the magnetic field and under the influence of a $0.5 \mathrm{~T}$ static magnetic field. Data in 

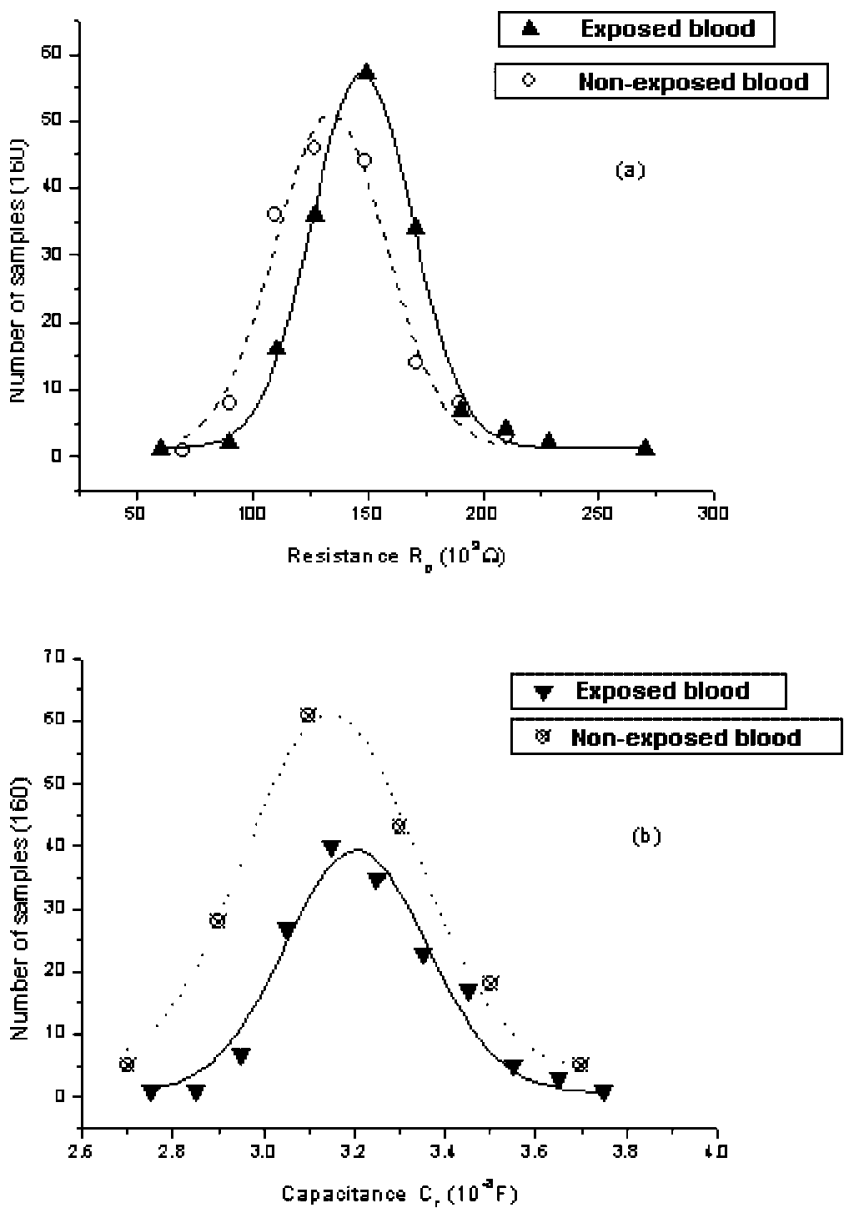

Fig. 3. Comparison between the distributions of blood electrical parameters. a: Electrical resistances. b: Capacitances. A total of 160 blood samples from healthy donors were used, both without the magnetic field and under the influence of a 0.5 Tstatic magnetic field. The continuous line corresponds to a Gaussian behavior. Data in this figure correspond to samples kept under magnetic field just during the time of measurement.

this figure correspond to samples kept under magnetic field just during the time of measurement. In Figure 3a, distributions of the electrical resistances $R_{\mathrm{p}}$ for samples both outside the magnetic field and under magnetic field exposure are presented. In this figure, we demonstrate that the resistances are shifted to the right one with respect to the other, while the widths are very similar. In Figure 3b, distributions for the capacitances $C_{\mathrm{T}}$ are shown. These two last distributions are more difficult to compare due to the data spreading. However, a slightly shift to the right is also observed when the magnetic field is present.

From these figures it is seen that the electrical parameters in both conditions follow a similar behavior. To determine the functional relation of the distributions, a fit was performed for the different cases. A Gaussian function corresponded to the best fit in all cases. It is important to stress that the fits shown in these figures roughly describe the data, but no other function was found to give a better description.

A Wilcoxon matched pairs statistical test was applied to both data sets. A statistically significant change was observed for both electrical blood parameters: resistance $(Z=5.06, P<0.001)$ and capacitance $(Z=3.32, P<0.001)$ after the magnetic field was applied.

In Table 1, the mean values for the measured blood electrical parameters are shown; the values obtained from our experiment change when the magnetic field was applied. Blood resistance increased by approximately $10.4 \%$, while the capacitance increased by $1.9 \%$. These percentage differences were determined by considering the shift in the mean values and normalizing with respect to the corresponding value without the magnetic field.

Table 2 shows measurements for five blood samples under different exposure conditions. First, the samples were measured immediately after applying the magnetic field. Then measurements were repeated after 15 min under magnetic field exposure and still in presence of the magnetic field, and finally $15 \mathrm{~min}$ after removing the magnets. In this last case the samples were first kept 15 min under magnetic field. In Table 2, the mean values for the electrical parameters change significantly under each condition. The resistance decreased when the field applied was off, while the capacitance seems to be oscillating.

The results of this investigation for the blood impedance have been described by a three-element circuit model, in agreement with the model previously proposed by Zhao et al. [1993]. However, it is important to note that our findings suggest that a CPE could be a better description for the system capacitance than a single capacitor.

On the other hand, significant differences on the $R_{\mathrm{p}}$ values were determined by measuring the impedance under the influence of the magnetic field or in its absence. On the contrary, in relation to the capacitance the changes observed were very small. A comparison between our results and those reported by Zhao [1993], establishes an important difference on the behavior of

TABLE 1. Mean $( \pm$ SD) Electrical Parameters for 160 Whole Human Blood Samples

\begin{tabular}{lcc}
\hline Blood sample & $\begin{array}{c}\text { Resistance } \\
R_{\mathrm{p}}\left(10^{3} \Omega\right)^{\mathrm{a}}\end{array}$ & $\begin{array}{c}\text { Capacitance } \\
C_{\mathrm{T}}\left(10^{-8} \mathrm{~F}\right)^{\mathrm{b}}\end{array}$ \\
\hline $\begin{array}{lcc}\text { Non-exposed to magnetic field } \\
\quad \text { Exposed to magnetic field }\end{array}$ & $134 \pm 2$ & $3.14 \pm 0.01$ \\
$\quad$ during measurement only & $149 \pm 1$ & $3.20 \pm 0.01$ \\
\hline
\end{tabular}

${ }^{\mathrm{a}} Z=5.06, P<0.001$ by Wilcoxon matched pairs test.

${ }^{\mathrm{b}} Z=3.32, P<0.001$. 
TABLE 2. Mean ( \pm SD) Electrical Parameters for Five Blood Samples

\begin{tabular}{lcc}
\hline Blood sample & Resistance $R_{\mathrm{p}}\left(10^{3} \Omega\right)$ & Capacitance $C_{\mathrm{T}}\left(10^{-8} \mathrm{~F}\right)$ \\
\hline Just after applying the magnetic field & $241 \pm 1$ & $2.82 \pm 0.01$ \\
After 15 min under magnetic field exposure & $241 \pm 1$ & $2.73 \pm 0.01$ \\
15 min after removing the magnetic field & $219 \pm 1$ & $2.92 \pm 0.01$ \\
\hline
\end{tabular}

blood capacitance. While we have not found important changes, they reported a significant variation on the capacitance value. A possible explanation for this apparent discrepancy could be the fact that they used four different anticoagulants and also an hematocrit range of $20 \%-60 \%$. In our case we use EDTA as anticoagulant in small concentrations (less than $1 \%$ in volume) and only samples in a narrower hematocrit concentration, from $40.4 \%$ to $54.8 \%$ were measured. A recent work by Jaspard and Nadi [2002] showed that EDTA used in small volumes does not modify the blood dielectric values.

\section{CONCLUSIONS}

Measurements of the electrical resistance and capacitance associated to the blood allowed the determination of changes supposedly attributed to the magnetic field. The mean values of these variables were determined by fitting a Gaussian function to the distributions. The mean electrical resistance $R_{\mathrm{p}}$ of blood was observed to increase from $(134 \pm 2) \times 10^{3} \Omega$ to $(149 \pm 2) \times 10^{3} \Omega$ when the sample was exposed to magnetic field, while the mean capacitance $C_{\mathrm{T}}$ changed from $(3.14 \pm 0.01) \times 10^{-8}$ to $(3.20 \pm 0.01) \times 10^{-8} \mathrm{~F}$ under magnetic field exposure. A Wilcoxon matched pairs statistical test was applied to determine the significance of these changes.

It is clear that several factors can affect this kind of experiments, such as the temperature, hematocrit concentration, interval time between measurements, coagulation, possible hemato-pathologies, etc. Some of them were avoided or at least diminished by measuring only samples from healthy donors that were clinical assessed before using. Measurements were performed at the same temperature conditions and anticoagulant was used. However, these factors must be considered carefully and require more research. Also, the physical mechanism that describes the influence of magnetic fields on blood remains unclear and further efforts are necessary for a better knowledge.

\section{ACKNOWLEDGMENTS}

The authors thank the technical support of the staff of the Centro Estatal de Transfusión Sanguínea at León, Guanajuato.

\section{REFERENCES}

Alison JM, Sheppard RJ. 1993. Dielectric properties of human blood at microwave frequencies. Phys Med Biol 38:971978.

Beving H, Eriksson G. 1994. Dielectric spectroscopy of human blood. Eur J Surg Suppl 574:87-89.

Beving H, Eriksson L, Davey CL, Kell DB. 1994. Dielectricproperties of human blood and erythrocytes at radio frequencies $(0.2-10-\mathrm{MHz})$ - dependence on cell-volume fraction and medium composition. Eur Biophysics J 23:207-215.

Capri M, Mesirca P, Remondini D, Carosella S, Pasi S, Castellani G, Franceschi C, Bersani F. 2004. $50 \mathrm{~Hz}$ sinusoidal magnetic fields do not affect human lymphocyte activation and proliferation in vitro. Phys Biol 1:211-219.

Coldman MF, Gent M, Good W. 1969. The osmotic fragility of mammalian erythrocytes in hypotonic solutions of sodium chloride. Comp Biochem Physiol 31:605-609.

Cole KS, Cole RH. 1941. Dispersion and absorption in dielectrics 1. Alternating current characteristics. J Chem Phys 9:341-351.

De Vries PMJM, Langendijk JWG, Kouw PM. 1995. The influence of alternating current frequency on flow related admittance changes of blood: A concept for improvement of impedance cardiography. Physiol Meas 16:63-69.

Gabriel C, Gabriel S, Corthout E. 1996a. The dielectric properties of biological tissues: I. Literature survey. Phys Med Biol 41:2231-2249.

Gabriel S, Lau RW, Gabriel C. 1996b. The dielectric properties of biological tissues: III. Parametric models for the dielectric spectrum of tissues. Phys Med Biol 41:2271-2293.

Grosse C. 1998. Permittivity of a suspension of charged spherical particles in electrolyte solution. 2. Influence of the surface conductivity and asymmetry of the electrolyte on the low and high-frequency relaxations. J Phys Chem 92:39053910.

Grosse C, Schwan HP. 1992. Cellular membrane potentials induced by alternating fields. Biophys J 63:1632-1642.

Hayashi Y, Livshits L, Caduff A, Feldman Y. 2003. Dielectric spectroscopy study of specific glucose influence on human erythrocyte membranes. J Phys D: Appl Phys 36:369-374.

Higashi T, Yamagishi A, Takeuchi T, Kawaguchi N, Sagawa S, Onishi S, Date M. 1993. Orientation of erythrocytes in a strong static magnetic field. J Blood 82:1328-1334.

Jaspard F, Nadi M. 2002. Dielectric properties of blood: An investigation of temperature dependence. Physiol Meas 23:547554.

Jaspard F, Nadi M, Rouane A. 2003. Dielectric properties of blood: An investigation of haematocrit dependence. Physiol Meas 24:137-147.

Lisin R, Ginzburg BZ, Schlesinger M, Feldman Y. 1996. Time domain dielectric spectroscopy study of human cells. I. Erythrocytes and ghosts. Biochemica et Biophysica Acta 1280:34-40.

McDonald JR. 1987. Linear relaxation: Distributions, thermal activation, structure, and ambiguity. J Appl Phys 62:R51R62. 
Nakano N, Ostuka J, Tasaki A. 1972. Paramagnetic anisotropy measurements on a single crystal of deoxyhemoglobin. Biochemica et Biophysica Acta 278:355-371.

Pauling L, Coryell C. 1936. The magnetic properties and structure of hemoglobin, oxyhemoglobin and carbonmonoxy hemoglobin. Proc National Acad Sci 22:210.

Polevaya Y, Ermolina I, Schlesinger M, Ginzburg BZ, Feldman Y. 1999. Time domain dielectric spectroscopy study of human cells. II. Normal and malignant white blood cells. Biochemica et Biophysica Acta 1419:257-271.

Poppendiek HF, Hody GL. 1963. The influence of alternating electric currents on haemolysis of human blood. Phys Med Biol 7:481-488.

Poppendiek HF, Hody GL, Greene ND, Glass JL, Hayes JR. 1964. In vivo study of the effects of alternating electric currents on some properties of blood in dogs. Phys Med Biol 9:215217.
Richert R. 1996. A simple current-to-voltage interface for dielectric relaxation measurements in the range $10^{-3} \mathrm{~Hz}$ to $10^{7} \mathrm{~Hz}$. Rev Sci Instrum 67:3217-3221.

Shalygin AN, Norina SB, Kondorsky EI. 1983. Behaviour of erythrocytes in high gradient magnetic field. J Magnetism Magn Mater 31:555-556.

Yamagishi A, Takeuchi T, Date M. 1992. Diamagnetic orientation of red blood cells in high magnetic fields. Physika 177B:523526.

Yamamoto T, Nagayama Y, Tamura M. 2004. A blood-oxygenationdependent increase in blood viscosity due to a static magnetic field. Phys Med Biol 49:3267-3277.

Zhao TX. 1993. Electrical impedance and haematocrit of human blood with various anticoagulants. Physiol Meas 14:299307.

Zhao TX, Jacobson B, Ribbe T. 1993. Triple-frequency method for measuring blood impedance. Physiol Meas 14:145-156. 